\title{
Some Simulation Results of the Put-Call Symmetry Method Applied to Stochastic Volatility Models
}

\author{
Yuri Imamura, Yuta Ishigaki, \\ Takuya Kawagoe, and Toshiki Okumura \\ Department of Mathematical Sciences, Ritsumeikan University \\ 1-1-1 Nojihigashi, Kusatsu, Shiga, Japan \\ Email: yuri.imamura@gmail.com
}

\begin{abstract}
This paper provides some results of numerical experiments of the Put-Call Symmetry method proposed by the authors in [4] under Heston and $\lambda$-SABR models.

Keywords: Barrier option, Stochastic volatility model, numerical method, Heston model, $\lambda$-SABR model
\end{abstract}

\section{Introduction}

The aim of the present paper is to give simulation results of the computation of the price of a single-barrier option under the two famous stochastic volatility models, namely Heston's [3] and $\lambda$-SABR models [5] by using the technique proposed in [4] which is referred to as "Put-Call Symmetry Method".

The notion "Put-Call Symmetry" is originally introduced in the context of (semi-) static hedging in [2]. Roughly, it means the equivalence in law of $X_{T}-X_{T \wedge \tau_{K}}$ and $X_{T \wedge \tau_{K}}-X_{T}$, where $\tau_{K}$ is the first hitting time of a stochastic process $X$ to $K \in \mathbb{R}$, which is a much weaker condition than the reflection principle. Carr and Lee [2] showed that a semi-static hedge using pathindependent option for a barrier option is possible if (a variant of) Put-Call Symmetry holds for the price process of the underlying. This in turn implies that the price of the barrier option is expressed by those of path-dependent (plain-vanilla) options. Our PutCall Symmetry method makes use of this property by "symmetrizing" the underlying price process. A more detailed explanation will be given in section 2 .

The simulation results will be presented in section 3. There, as a benchmark, we simulate the path of the underlying price process by the Euler-Maruyama scheme, and we calculate the price by using MonteCarlo simulation. Then we compare it with our results of the new scheme. The results in this report may imply that our scheme requires less time partitions than the path simulation method.

\section{The Put-Call Symmetry Method}

\subsection{Arithmetic Put-Call Symmetry}

Let $(\Omega, \mathcal{F}, \mathbf{P})$ be a complete probability space with a right-continuous filtration $\left\{\mathcal{F}_{t}\right\}$ such that $\mathcal{F}_{0}$ contains all null sets of $\mathcal{F}$. Let $X$ be a real valued Markov process defined on $\left(\Omega, \mathcal{F}, \mathbf{P},\left\{\mathcal{F}_{t}\right\}\right)$. Following the terminology in [2], we say that arithmetic put-call symmetry (APCS) at $K$ holds for $X$ if

$$
\mathbf{E}\left[G\left(X_{t}-K\right) \mid X_{0}=K\right]=\mathbf{E}\left[G\left(K-X_{t}\right) \mid X_{0}=K\right]
$$

holds for any bounded measurable function $G$. The APCS means that $X_{t}$ has the same distribution as $X_{t \wedge \tau_{K}}-\left(X_{t}-X_{t \wedge \tau_{K}}\right)$ for $t \geq 0$.

Proposition 2.1 (Carr-Lee [2] Theorem 3.1, [1], [4]). Let $X$ be given by the following one-dimensional stochastic differential equation:

$$
d X_{t}=\sigma\left(X_{t}\right) d W_{t}+\mu\left(X_{t}\right) d t
$$

where $\sigma$ and $\mu$ imply uniqueness in law for equation (1). Fix $K \in \mathbb{R}$. Suppose that

$$
\begin{gathered}
\sigma(K+x)=\sigma(K-x) \\
\mu(K+x)=-\mu(K-x)
\end{gathered}
$$

for any $x \in \mathbb{R}$. Then APCS at $K$ holds for $X$.

In [2], Proposition 2.1 applied to the pricing and semistatic hedging of barrier options. "Semi-static hedging" here means replication of the barrier contract by trading European-style claims at no more than two times after inception. This means a semi-static hedging strategy reduces transaction costs dramatically than the standard dynamic hedging one.

Precisely speaking, we have the following: if $X$ satisfies APCS at $K \in \mathbb{R}$, then for any bounded measurable $f$ and $T>0$,

$$
\begin{aligned}
\mathbf{E}\left[f\left(X_{T}\right) I_{\left\{\tau_{K}>T\right\}}\right]=\mathbf{E}[ & \left.f\left(X_{T}\right) I_{\left\{X_{T}<K\right\}}\right] \\
& -\mathbf{E}\left[f\left(2 K-X_{T}\right) I_{\left\{X_{T}>K\right\}}\right],
\end{aligned}
$$

where $\tau_{K}:=\inf \left\{t>0 ; X_{t} \geq K\right\}$. The left-hand-side reads the price of a barrier option knocked out at $K$, and the right-hand-side is the price of a combination of two plain-vanilla options. 


\subsection{Construction of the Symmetrized Diffusion}

In [4], we give a new perspective to the above framework. Here we briefly recall the essential ideas.

We start with a general (one-dimensional) diffusion process $X$ satisfying

$$
d X_{t}=\sigma\left(X_{t}\right) d W_{t}+\mu\left(X_{t}\right) d t
$$

where $\sigma$ and $\mu$ imply uniqueness in law for equation (5), without the symmetric condition (2) and (3). We then construct another diffusion ("shadow path") $\tilde{X}$ that satisfies APCS at $K$ in the following way. Put

$$
\begin{gathered}
\tilde{\sigma}(x):= \begin{cases}\sigma(x) & x<K \\
\sigma(2 K-x) & x \geq K,\end{cases} \\
\tilde{\mu}(x):= \begin{cases}\mu(x) & x<K \\
-\mu(2 K-x) & x \geq K,\end{cases}
\end{gathered}
$$

and define $\tilde{X}_{t}$ as a (weak) solution to

$$
d \tilde{X}_{t}=\tilde{\sigma}\left(\tilde{X}_{t}\right) d W_{t}+\tilde{\mu}\left(\tilde{X}_{t}\right) d t
$$

Then $\tilde{\sigma}$ and $\tilde{\mu}$ satisfies the conditions (2) and (3), therefore $\tilde{X}$ satisfies APCS at $K$ by Proposition 2.1. Then we retrieve (4); namely we have

$$
\begin{aligned}
\mathbf{E}\left[f\left(\tilde{X}_{T}\right) I_{\left\{\tau_{K}>T\right\}}\right]=\mathbf{E}[ & \left.f\left(\tilde{X}_{T}\right) I_{\left\{\tilde{X}_{T}<K\right\}}\right] \\
& -\mathbf{E}\left[f\left(2 K-\tilde{X}_{T}\right) I_{\left\{\tilde{X}_{T}>K\right\}}\right] .
\end{aligned}
$$

The key observation to our new framework is the following:

$$
\mathbf{E}\left[f\left(\tilde{X}_{T}\right) I_{\left\{\tau_{K}>T\right\}}\right]=\mathbf{E}\left[f\left(X_{T}\right) I_{\left\{\tau_{K}>T\right\}}\right] .
$$

In fact, for $T \leq \tau_{K}, X_{T}$ is equal to $\tilde{X}_{T}$ pathwisely. We then have a new equation:

$$
\begin{aligned}
\mathbf{E}\left[f\left(X_{T}\right) I_{\left\{\tau_{K}>T\right\}}\right]=\mathbf{E}[ & \left.f\left(\tilde{X}_{T}\right) I_{\left\{\tilde{X}_{T}<K\right\}}\right] \\
& -\mathbf{E}\left[f\left(2 K-\tilde{X}_{T}\right) I_{\left\{\tilde{X}_{T}>K\right\}}\right]
\end{aligned}
$$

We do not anymore regard (7) as an equation for semi-static hedging but a relation to give a numerical scheme to calculate the expectation of the diffusion with Dirichlet boundary condition in terms of those without boundary conditions. The former is very difficult while the latter is rather easier using rapidly developing technique from numerical finance.

\section{$3 \quad$ Numerical Results}

This section provides numerical examples of our new scheme for pricing barrier options with barrier $K$ under Heston model and $\lambda$-SABR model. Since they are two-dimensional models, the above framework is not directly applicable. But we can nonetheless rely on our method thanks to the following result from [1]:
Proposition 3.1 ([1]). Let $X$ be given by the following stochastic differential equation:

$$
\left\{\begin{array}{l}
d X_{t}=\sigma\left(X_{t}, V_{t}\right) d W_{t}^{1}, \\
d V_{t}=\alpha\left(V_{t}\right) d t+\beta\left(V_{t}\right)\left(\rho d W_{t}^{1}+\sqrt{1-\rho^{2}} d W_{t}^{2}\right),
\end{array}\right.
$$

where $\sigma, \alpha$ and $\beta$ imply uniqueness in law, $\left(W_{t}^{1}, W_{t}^{2}\right)_{t \geq 0}$ is a 2-dimensional Brownian motion and $\rho$ is a constant $\in[0,1]$. If $\sigma(\cdot, V)$ satisfies (2) for any $V$, then $X$ has the APCS.

Here we assume that the payoff is call option with strike $U$, that is, we estimate

$$
\begin{aligned}
& A= \mathbf{E}\left[\left(\tilde{X}_{T}-U\right) I_{\left\{\tilde{X}_{T}<K\right\}}\right] \\
& \quad-\mathbf{E}\left[\left(2 K-\tilde{X}_{T}-U\right) I_{\left\{\tilde{X}_{T}>K\right\}}\right], \\
& B=\mathbf{E}\left[\left(X_{T}-U\right)^{+} I_{\left\{\tau_{K}>T\right\}}\right] .
\end{aligned}
$$

As a benchmark, we also give numerical results by a simple application of Monte-Carlo simulation to EulerMaruyama approximation of the diffusion paths, where we regard the pay-off of barrier options as a function of the whole path.

\subsection{Heston Model}

First, we deal with Heston model, where the underlying price process is given as follows:

$$
\left\{\begin{aligned}
d X_{t}= & r X_{t} d t+\sqrt{\sigma_{t}} X_{t} d W_{t}^{1} \\
d \sigma_{t}= & \kappa\left(\theta-\sigma_{t}\right) d t \\
& \quad+\nu \sqrt{\sigma_{t}}\left(\rho d W_{t}^{1}+\sqrt{1-\rho^{2}} d W_{t}^{2}\right) .
\end{aligned}\right.
$$

Here $\left(W_{t}^{1}, W_{t}^{2}\right)_{t \geq 0}$ denotes a 2-dimensional Brownian motion. Then the shadow path $\tilde{X}$ is satisfied the following equation:

$$
\left\{\begin{aligned}
d \tilde{X}_{t}= & \left(I_{\left\{\tilde{X}_{t}<K\right\}} \tilde{X}_{t}+I_{\left\{\tilde{X}_{t} \geq K\right\}}\left(2 K-\tilde{X}_{t}\right)\right) \sqrt{\sigma_{t}} d W_{t}^{1} \\
& +\left(I_{\left\{\tilde{X}_{t}<K\right\}} \tilde{X}_{t}-I_{\left\{\tilde{X}_{t} \geq K\right\}}\left(2 K-\tilde{X}_{t}\right)\right) r d t \\
d \sigma_{t}= & \kappa\left(\theta-\sigma_{t}\right) d t \\
& +\nu \sqrt{\sigma_{t}}\left(\rho d W_{t}^{1}+\sqrt{1-\rho^{2}} d W_{t}^{2}\right) .
\end{aligned}\right.
$$

In the simulations, the parameters are set to be the following: The initial underlying asset's price and variance are $X_{0}=100$ and $\sigma_{0}=0.03$, respectively. The mean reversion speed and level are set to be $\kappa=1$ and $\theta=0.03$, respectively. The barrier is set to be $K=120$. The correlation rate is set to be $\rho=-0.7$, and we set $\nu=0.03$. In this paper, we set the interest rate $r$ to be 0 . We set the strike price to $U=100$.

We give results of a simulation where the number of time-partition is 10 to 100 in Euler-Maruyama approximation and the number of times in Monte-Carlo simulation is cube of the number of time-partition.

In the benchmark of path-Monte Carlo simulation method, the number of partition is 5,000 and that of simulation is $10,000,000$. The result is 1.6101388251 . 


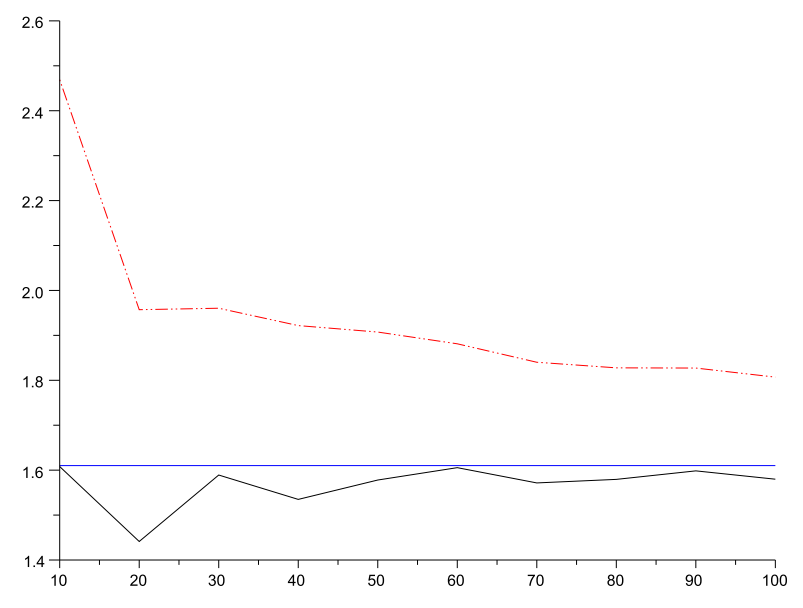

Fig. 1: Simulation Result under Heston model ( $\mathrm{r}=0)$
Table 1: Heston model $(\mathrm{r}=0)$

\begin{tabular}{|c|c|c|c|c|}
\hline Time Partition & $\mathrm{A}$ & $\mathrm{B}$ & Error(A) & Error(B) \\
\hline \hline 10 & 1.6079 & 2.4689 & 0.1334 & 53.3398 \\
\hline 20 & 1.4412 & 1.9571 & 10.4898 & 21.5516 \\
\hline 30 & 1.5891 & 1.9604 & 1.3041 & 21.7538 \\
\hline 40 & 1.5349 & 1.9217 & 4.6719 & 19.3539 \\
\hline 50 & 1.5780 & 1.9073 & 1.9947 & 18.4603 \\
\hline 60 & 1.6055 & 1.8812 & 0.2855 & 16.8359 \\
\hline 70 & 1.5715 & 1.8401 & 2.3956 & 14.2862 \\
\hline 80 & 1.5794 & 1.8277 & 1.9047 & 13.5128 \\
\hline 90 & 1.5984 & 1.8272 & 0.7285 & 13.4838 \\
\hline 100 & 1.5798 & 1.8070 & 1.8795 & 12.2293 \\
\hline
\end{tabular}

The simulation results of our scheme are shown in Figure 1 and Table 1 below. In Figure 1, the solid line is a result of our scheme method; $A$ in (8), and the dotted line is the one of path Monte-Carlo simulation; $B$ in (8). In Table 1, a result of our scheme, the error between our scheme and the benchmark, a result of path MonteCarlo, the error between the path Monte-Carlo result and the benchmark, respectively, are shown. Here the error between the results and the benchmark are given by

$$
\left|\frac{A_{N}-\text { the benchmark }}{\text { the benchmark }}\right|,
$$

and

$$
\left|\frac{B_{N}-\text { the benchmark }}{\text { the benchmark }}\right|,
$$

where $\left(A_{N}\right)_{N=1}^{10000}, \quad\left(B_{N}\right)_{N=1}^{10000}$ are the results of our scheme $A$ and the one of $B$ with $N$ times Monte-Carlo simulation, respectively.

\section{$3.2 \lambda$-SABR Model}

The next numerical example is based on $\lambda$-SABR model which is described as

$$
\left\{\begin{aligned}
d X_{t}= & r X_{t} d t+\sigma_{t} X_{t}^{\beta} d W_{t}^{1} \\
d \sigma_{t}=\lambda & \left(\theta-\sigma_{t}\right) d t \\
& \quad+\nu \sigma_{t}\left(\rho d W_{t}^{1}+\sqrt{1-\rho^{2}} d W_{t}^{2}\right) .
\end{aligned}\right.
$$

Then the shadow path $\tilde{X}$ is given by the following SDE:

$$
\left\{\begin{aligned}
d \tilde{X}_{t}= & \left(I_{\left\{\tilde{X}_{t}<K\right\}} \tilde{X}_{t}^{\beta}+I_{\left\{\tilde{X}_{t} \geq K\right\}}\left(2 K-\tilde{X}_{t}\right)^{\beta}\right) \sigma_{t} d W_{t}^{1} \\
& +\left(I_{\left\{\tilde{X}_{t}<K\right\}} \tilde{X}_{t}-I_{\left\{\tilde{X}_{t} \geq K\right\}}\left(2 K-\tilde{X}_{t}\right)\right) r d t \\
d \sigma_{t}= & \lambda\left(\theta-\sigma_{t}\right) d t \\
& +\nu \sigma_{t}\left(\rho d W_{t}^{1}+\sqrt{1-\rho^{2}} d W_{t}^{2}\right) .
\end{aligned}\right.
$$

We consider the cases of $\beta=0.8$. The parameters are set to be the following: The initial underlying asset's price is $X_{0}=100$. The initial volatility is $\sigma_{0}=0.3$. The mean reversion speed and level are set to be $\lambda=1$, $\theta=0.3$. The barrier is set to be $K=120$. Also we set $\rho=-0.7$ and $\nu=0.3$. The riskless interest rate $r$ is set to be 0 . We set the stock price to $U=100$.

We give results where the numbers of time-partition and simulation time are the same as those in the previous section. The benchmark result is 3.1447152809 , where the number of partition is 5,000 and the number of the simulations is 10,000, 000 .

The results are shown in Figure 2 and Table 2 below. our scheme method, and the dotted line is the one of Monte-Carlo simulation. In Table 2, the result of our scheme, the error between our scheme and the benchmark, a result of path Monte-Carlo, the error between path Monte-Carlo and the benchmark are denoted.

Remark 3.2. Since we let $r=0(\Longleftrightarrow$ drift part $=0)$, the symmetrization (6) is applied only for $\sigma$. This made our simulations much easier. We note that our scheme will also work even when $r \neq 0$. 


\section{Conclusion}

From these results, we may conclude that our scheme is more effective than the path simulation method. For detailed discussion, see [1] and [4].

\section{References}

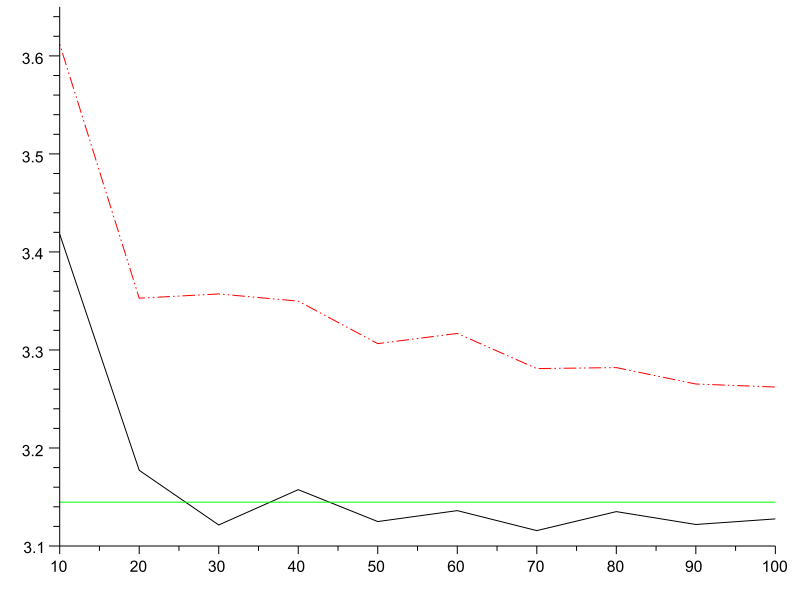

Fig. 2: Simulation Result under $\lambda$-SABR model $(\mathrm{r}=0)$

[1] Akahori, J. and Imamura, Y. (2012) "On a Symmetrization of Duffusion Processes", preprint.

[2] Carr, P., and R. Lee (2009): "Put-Call Symmetry: Extensions and Applications", Mathematical Finance, 19(4), 523-560.

[3] Heston, SL., (1993): "A Closed-Form Solution for Options with Stochastic Volatility with Applications to Bond and Currency options", Review of Financial Studies, 6 (2), 327-343.

[4] Imamura, Y., Y. Ishigaki, T. Kawagoe, and T. Okumura (2012): "A Numerical Scheme Based on Semi-Static Hedging Strategy", preprint.

[5] Henry-Labordere, P., (2005): "A General Asymptotic Implied Volatility for Stochastic Volatility Models", Available at SSRN: http://ssrn.com/abstract $=698601$.

Table 2: $\lambda$-SABR model $(\mathrm{r}=0)$

\begin{tabular}{|c|c|c|c|c|}
\hline Time Partition & $\mathrm{A}$ & $\mathrm{B}$ & Error(A) & Error(B) \\
\hline \hline 10 & 3.4181 & 3.6119 & 8.6947 & 14.8584 \\
\hline 20 & 3.1772 & 3.3528 & 1.0337 & 6.6172 \\
\hline 30 & 3.1214 & 3.3571 & 0.7393 & 6.7552 \\
\hline 40 & 3.1574 & 3.3498 & 0.4058 & 6.5223 \\
\hline 50 & 3.1249 & 3.3064 & 0.6288 & 5.1434 \\
\hline 60 & 3.1361 & 3.3168 & 0.2738 & 5.4742 \\
\hline 70 & 3.1157 & 3.2810 & 0.9222 & 4.3338 \\
\hline 80 & 3.1350 & 3.2820 & 0.3062 & 4.3657 \\
\hline 90 & 3.1219 & 3.2652 & 0.7237 & 3.8337 \\
\hline 100 & 3.1276 & 3.2621 & 0.5425 & 3.7346 \\
\hline
\end{tabular}

